\title{
Simplicity and Complexity in Metaphysics
}

\author{
Aleksandr Kulieshov ${ }^{1}$ \\ ${ }^{1}$ Cherkasy State Technological University \\ 460 Shevchenko Boulevard, 18006, Cherkasy, Ukraine
}

DOI: $10.22178 /$ pos.35-3

LCC Subject Category:

BD95-131

Received 10.05.2018

Accepted 10.06.2018

Published online 17.06.2018

Corresponding Author:

klshv2014@gmail.com

(C) 2018 The Author. This

article is licensed under a

Creative Commons

Attribution 4.0 License

(c) (1)

\begin{abstract}
The article deals with the problem of simple and complex objects in metaphysical research. It is stated that the ultimate simplification of reality theoretical models follows from the nature of metaphysical knowledge. The modern metaphysical conceptions of fundamental simplicity and fundamental complexity of existing objects are discussed. They are the conceptions of full and distributional monism, mereological nihilism, and the traditional binary or binary-based models of the foundations of reality, ascending to Aristotle. The paper shows that these conceptions and ideas do not simplify reality as much as possible. The problematic and ambiguous nature of the conceptions considered is that all complicating factors are not excluded in them. This leads to a situation where further grounding of the entities that are accepted as the simplest ones is required. The state of the full simplification in metaphysics is defined. It is argued that ultimate simplification requires the exclusion of qualitative multiplicity and quantitative definiteness, the exclusion of the distinction between substance and its qualities, between the entity and the law, the relation or condition which ground it. The entity identified in the process of ultimate simplification must be quantitatively indefinite, identical to its quality and substratum, indistinguishable from the law, the condition or the relationship of its grounding. Such an entity can be the starting point for the construction of a metaphysical model of the world as a consistent complication of entities.
\end{abstract}

Keywords: metaphysics; entity; simplicity; complexity; fundamental reality; grounding.

\section{INTRODUCTION}

Explaining reality in which we find ourselves, we intuitively seek for simplicity. It seems natural that simple ideas are more plausible than complex ones. The more complex reality commonly requires the greater volume and complexity of explanation. Before the searching mind there appears a frightening specter of infinite complication. The simple, on the contrary, is undoubtedly certain, clear, foreseeable. The problem of finding simple grounds is especially acute when one explains the world as a whole which is the task of metaphysics.

The contradictions that arise in this case seem to have a general epistemological character, since they refer equally to metaphysics, and to special sciences. Both modern special sciences and modern metaphysics are in search of simple explanations of complex phenomena. The results that we observe, however, are opposite to what one is looking for. This is most clearly manifested in modern natural sciences. Generally speaking, the natural-science theories are becoming more and more complicated. Explanations are often hard to grasp because of abundance and ambiguity of their details. The coordination of these details becomes a special and increasingly complex task. If a way out of this situation is possible, then, most likely, it will be realized by means of metaphysics. Whatever scientific questions we answer, they imply a preliminary answer to the metaphysical question: so is the world basically simple or infinitely complex? 
In modern metaphysics this problem is undoubtedly realized, and attempts are being made to solve it. Moreover, it can be assessed as one of the central points in metaphysical discourse. The problem of simplicity is actively discussed in analytical metaphysics. Certain approaches, the principles of its investigation have been worked out so far. First of all, it is necessary to take into account the division of simplicity into two kinds: ontological and ideological, accepted by metaphysicians of the analytical tradition. Ontological simplicity (and accordingly, ontological complexity) refers to simple entities, ideological simplicity - to simple concepts.

In other words, this is the distinction between things in the world and concepts needed to describe the world [1, p. 16]. S. Cowling represents it as the two kinds of theoretical parsimony: ontological parsimony, which concerns the minimizing of ontological commitments, and ideological parsimony, which concerns the minimizing of ideological commitments [2, p. 3994].

Another pair of simplicity markers in metaphysics is quantitative and qualitative simplicity. It may also be expressed in terms of theoretical parsimony. Quantitative parsimony concerns the number of commitments while qualitative parsimony concerns the number of kinds of commitments [2, p. 3996]. In other words, quantitative parsimony, as it is defined by S. Z. Elgin, involves the number of entities a theory posits; qualitative parsimony involves the number of kinds of entities a theory posits [3, p. 3]. In both cases it goes about minimal quantity: in one case - the minimal quantity of primitive entities in the world; in another case - the minimal quantity of primitive kinds of entities.

No understanding of these points of view is possible without the answers to some principal questions about the nature of simplicity and complexity as well as about their relations. To analyze the problem we need to know, first of all, whether it make sense to talk about simplicity and complexity? Is there both ontological and ideological simplicity or only one of them is worth deliberation? Is there something ultimately simple in our world? Is there something complex in the world? Is everything complex reducible to simple? Are complex entities formed by the simple ones? Accordingly, is it possible to build a metaphysical model of the world, based on ultimately simple entities?
The aim of the paper is to reveal the place of simplicity and complexity in metaphysics, answering the posed questions. To do this, we need to analyze the existing metaphysical conceptions of simplicity and complexity.

\section{RESULTS AND DISCUSSION}

The complexity of accessible to us reality should be regarded as something empirically given. Even denying any complexity from the Eleatic position, it is necessary to explain this empirical complexity. At least in the form of an illusion, complexity exists, and there must be some explication of the fact. There must be a ground of complexity which may be itself something complex or simple.

Simplicity is less obvious. It is natural to doubt simplicity suspecting it to hide some implicit complexity. It is simplicity that looks more like an illusion. Disbelief in simplicity may be the matter of scientific intuition as in these words of T. Parsons (1976): "There is no prima facie reason to suppose that the universe contains a small number of things, or a small number of kinds of things. There is no prima facie reason to believe that a theory that endorses a smaller number of things, or kinds of things, or employs a smaller number of primitives, is simpler or likelier to be true or likely to yield more insight than another" [4, p. 660]. One more way of arguing against simplicity is to refute initially plausible justifications for simplicity in metaphysics [5]. More sophisticated anti-simplicity position is based on understanding that what is simple from one perspective is complex from another [6]. Thus, everything simple in a certain sense is complex and vice versa. If such is the state of affairs, it removes the question of something absolutely simple or absolutely complex as the ground for what exists. The very coexistence of simplicity and complexity in one is something complex. So the world is infinitely complex from this point of view.

Are there any counterarguments refuting the proposed decision? One can say that reality, which is only complex, requires a ground of its complexity, it cannot be ungrounded. Any complexity appeals for the answer to the question: why is it so complex? It follows that no complex entity can be the ultimate ground of existing totality. Either the world lacks absolutely simple entities or there is no unique ground for everything that exists. Only one of these alternatives 
can be real. Assuming, as an axiom, the existence of an ultimate ground we conclude that there are not only complex entities in the world. There is something simpler and something more complicated in absolute sense. The absolutely simple entity (if such entity or entities exist) is not complex in a different sense.

The idea that there are fundamental entities and their derivatives has sufficient support in modern metaphysics. This idea characterize what is called (according to F. Jackson's words) the serious metaphysics. Three principles of the serious metaphysics are formulated by U. Kriegel. They are completeness ("Serious metaphysics attempts to produce a total theory of the world, that is, a theory of everything"), discrimination ("Serious metaphysics discriminates between fundamental and derivative statements and notions"), and entailment ("The relationship between fundamental and derivative statements in the total theory of the world is that of entailment") [7. p. 17]. By words of J. L. Dowell the serious metaphysics is an attempt to say what the world is like in terms of a limited number of more or less basic notions [8, p. 91]. It is apparent that the principle of fundamentality is formulated here in "ideological" not "ontological" terms. But it doesn't exclude ontological interpretation. The main problem as for metaphysical simplicity is in getting to know whether the simple is fundamental or, differently, whether the fundamental is simple.

The conception that there are fundamental entities and derived entities is related to simplicity and complexity. If fundamental entities exist, then they should be simpler than nonfundamental entities. If simple entities are fundamental, then on their basis all other (nonfundamental) entities are constructed. It follows from the premise of fundamentality. Thus, we have the ground to regard in metaphysical perspective the complexity as a complication of the simple. On the other hand, in the same perspective, a complex entity can be reduced to a simple one (or some simple ones). Simplification (at least in some cases) is equivalent to the fundamentalization of entities. This principle successfully works in many theoretical constructions. At the same time, no theory can avoid the use of conceptual primitives.

Does it mean that metaphysical simplicity is only a theoretical (or ideological) phenomenon? We will find a positive answer to this question in descriptive metaphysics - the trend in analytical philosophy, which goes back to the works of P. Strawson. The basic principle of descriptive metaphysics lies in the conviction that we are not able to go beyond our ideas about the world, everything that we are discussing is related to the so-called conceptual scheme. Only the existing system of concepts is the real subject of discussion and description in metaphysics. From this point of view, we do not know how complex reality is, but its description can be simple.

But such a position is not correct. First, we can distinguish and contrast the knowledge of the world and the knowledge of our knowledge of the world. This opposition distinguishes in knowledge the part which is directly related to reality. Secondly, in any case, ideological simplicity is impossible without the ontological one. If simple concepts correspond to reality, then ideological simplicity coincides with ontological simplicity. If simple concepts do not correspond to reality, then the knowledge of ontologically simple entities (as far as it possible) should be preferred. Therefore, further on, only ontological simplicity will be discussed.

How can something extremely simple look like in terms of quantitative and qualitative characteristics? Firstly, it can be something substantially one and of unique quality. Secondly, it can be something substantially one and of multiple qualities. Thirdly, it can be something substantially multiple and of unique quality. Fourthly, it can be something substantially multiple and of multiple qualities. Three of these four possibilities are reflected in modern metaphysical conceptions.

Let the first of the four conceptions be called "the full monism". It generally coincides with the wellknown classical conception of the Eleatics. Anything complex is excluded from this conception as there is no possible transition from qualitatively and quantitatively unique entity to complex entities. So one can infer only that empirical complexity is an illusion. This situation doesn't look plausible just because of the presence of illusion and the subject possessing it. The subject and the subject's illusions make the situation more complicated than it is acceptable for the full monism.

The term "monism" in the nowadays metaphysics often mark the conception of a unique multiqualitative reality. According to D. M. Cornell, it refers to the view that there is only a single material object in existence: the world itself. The world is mereologically simple, it has no proper 
parts [9, p. 2398]. The world, as a whole, has certain properties which give rise to the appearance of there being a multiplicity of material things. These properties D. M. Cornell call "distributional" as they ascribe to an object the particular way in which that quality is distributed across the extension of that object [9, p. 2405]. So it is natural to define the second of our possible metaphysical conceptions of fundamental simplicity-complexity as "the distributional monism". Another defender of this one category ontology L. A. Paul finds its advantage in that it collapses the distinction between particular and property, replacing it with a single fundamental category of intrinsic characters or qualities [10, p. 32]. So it is the world where there are no entities apart from qualities and their distribution. The picture seems plausible but leaves some doubt as for its absolute simplicity. What properties are simple and why exactly these ones, why they are distributed this way and not another - is left without explanation. One may suppose that the layer of reality consisting of distributional properties is not fundamental. It needs further grounding.

The third variant of fundamental simplicity is represented by the so called "mereological nihilism". In general form it is defined by T. Sider as the conception according to which composite entities (entities with proper parts) do not exist [11, p. 237]. There exist only simple entities and their compositions. So there are "no chairs or tables, no rocks or planets, no planes, trains or automobiles" but there are "simples arranged chair-wise, rock-wise, and so on and so forth" [9, p. 2402]. Mereological simples apparently may have one and the same property or they may have different properties. Let's take one property simples. If such property does not fully coincide with a primitive fundamental entity, then the simplest entity appears to be in a sense complex which makes the whole picture questionable in the aspect of simplicity. If, on the contrary, there is coincidence of the stated property and an entity, then the question of the simples' multiplicity arises. Why are there so many quantitatively different objects in the fundamental layer of reality? This must have its ground which is to be made clear.

The conception of mereological nihilism may be weakened by acceptance of some complex objects being in a sense primitive i. e. having properties not found in the fundamental layer of reality. One such weakened nihilism conception be- longs to P. van Inwagen who adds to physical simples living organisms as the second kind of real entities [12]. A more successive conception of that sort is called universalism. According to it any objects compose a further object. K. Miller discerns two types of universalism: generative universalism and non-generative universalism. The first presupposes that "for any set of particulars there exists something that is composed of those particulars such that the composite is not identical to any one of its parts or to its parts considered collectively". The second assumes that there are also "non-generative composites: composite objects that are identical to the totality of their parts or to the totality of their parts arranged in a certain way" [13, pp. 533-534]. Does this change the situation with primitive simples? Not much. New properties that arise in complex objects and new objects that look like ontological primitives increase the scope of fundamental entities and fundamental qualities in need of grounding. The problem becomes more complicated and tangled. But its essence remains the same: the objects which are regarded as ultimately simple and fundamental are not really such. They are not ungrounded or self-grounded if there are generally grounding relations in the world.

One can imagine fundamental essences that are extremely simple but diverse, differing from each other quantitatively and qualitatively. This is the fourth of the above options of fundamental simplicity. The most popular are the traditional binary or binary-based models of the foundations of reality, ascending to Aristotle. A modern variant of such models based on Aristotle's Categories is the so called "Ontological Square", a fourfold division of entities arising from two orthogonal distinctions: 1 . attributes vs. substances; 2. universals vs. particulars. So we have as a result of cross-wise combination a categorical scheme which comprises universal and particular substances, i. e. kinds and objects, as well as universal and particular attributes, i. e. characters and moments [14, p. 27]. This list of four categories exhausts the simplest entities. It makes an impression of logical completeness and besides it corresponds to our ordinary representations expressed in speech practice. But is this enough? It should be noted that the selected categories are not particulars. These are species that imply splitting into individual entities. Such a partition is not specified by the species themselves. It requires additional grounding, and this 
circumstance makes the fundamental categories not extremely simple, but rather complex, therefore, not fundamental.

In any of the versions of fundamental simplicity it appears necessary to have some addition in the form of a law, a condition or a grounding relation which make these entities the simplest ones. It seems that this addition gives an opportunity of a decisive choice in the process of simplification or otherwise - complication of reality. Some metaphysicians propose evaluating theoretical simplicity to take into account the number and the complexity of laws cited by a theory [15], the circumstances under which composition of entities occurs [13], the simplicity of grounding relations [3]. These considerations, nevertheless, do not remove further questions. Where do these laws, conditions, relations come from? If they are completely conditioned by the simplest entities that correlate, they do not add anything to the problem of grounding these entities. If they are not conditioned by the simplest entities, then they require their separate grounding as well as general grounding for them and those entities they unite. In other words they require simplification.

A possible answer to the situation is the concept of brutalism, based on the assertion that the conditions for composing entities are a brute fact, which should not be further analyzed. According to N. Markosian whenever composition occurs or whenever it fails to occur, it is just a "brute fact" [16, pp. 4-5]. The concept of brutalism means in fact the total refusal to explain. Alternatively the explanation is shifted to entities themselves, both simple and complex, which, in turn, requires explanations. Such a decision is hardly satisfactory from a metaphysical point of view.

Does the review of contemporary theoretical models bring us closer to an understanding of simplicity and complexity in metaphysics? If we comprehend metaphysics as an extremely abstract and general knowledge of the world, we have the clue to some positive decisions about simplicity and complexity. The ultimate abstractness of metaphysical entities corresponds to their ultimate simplicity.

What does the ultimate simplification of the objects of metaphysical consideration mean? To do this, it is necessary to exclude all the complicating factors, if possible, of course. If not, to explain why. The problematic and ambiguous nature of the conceptions considered is that all complicating factors are not excluded in them. This leads to a situation where further grounding of the entities that are accepted as the simplest ones is required. As a result, we have not the ultimate picture of what exists, but an empirically concrete one.

To simplify the subject of research as much as possible, it is first of all necessary to exclude the ideological layer of description, to turn to the subject itself as an objective reality. Further, quantitative and qualitative multiplicity should be excluded. It is quite obvious that the simplest cannot be different, either quantitatively or in respect of different kinds. It may seem puzzling how the unique entity divide itself into the multiplicity of empirically fixed individual entities. However, the point is that the simplest should not be regarded as one entity in the arithmetic sense. In other words, the simplest should not be considered as the one. It just means that the ultimately simple has no quantitative definiteness. Quantitative definiteness complicates existence. The simplest entity must be out of quantity, this is something that cannot be counted.

Naturally, one should exclude the difference between the property and the substance. This difference also complicates things. The simplest is that which is both the quality and the carrier of this quality. Moreover, the quality and the carrier in it are indistinguishable (they become discernible only if the simplest entity becomes more complicated). Finally, maximal simplification means excluding the difference between the entity and its grounding. The law, relation or condition that sets the entity of the simplest kind should not be distinguished from the entity itself. The simplest entity and the law or condition of its existence, the relation that determines it should be the same. In other words, the entity must be for itself an action that causes its complication.

If we have found such a simple entity, we have found the foundation of everything that exists in the world. First of all, it does not need grounding. At the same time, it is the ground for the existence of more complex entities, including things, properties, relationships, different forms of determinism. It is possible to construct a metaphysical picture of the world, using the simplest entity as a ground, the same entity as a grounding relation, and again this entity as something grounded. Only in this case, metaphysics will be completely "serious", representing the process of constructing reality by successive complication the ultimately simple entity. 


\section{CONCLUSION}

The problem of complexity and simplicity of the existing is central in metaphysics. The desire for ultimate simplification of reality follows from the fundamental nature of metaphysical knowledge. Modern metaphysics carries out a search in this direction, relying on the guidelines of ontological and ideological simplicity, as well as quantitative and qualitative simplicity. The conceptions of fundamental simplicity and fundamental complexity of the existing are discussed. Ideas of quantitative and qualitative monism or pluralism of the simplest entities are advanced. The paper shows that these conceptions and ideas do not simplify the reality as much as possible. Because of this, the identified simplest entities require further grounding. Ultimate simplification requires the exclusion of qualitative multiplicity and quantitative definiteness, the exclusion of the distinction between substance and its qualities, between the entity and the law, the relation or condition which ground it. The entity identified in the process of ultimate simplification must be quantitatively indefinite, identical to its quality and substratum, indistinguishable from the law, the condition or the relationship of its grounding. Such an entity can be the starting point for the construction of a metaphysical model of the world as a consistent complication of entities.

\section{REFERENCES}

1. Bradley, D. (2017). Philosophers should prefer simpler theories. Philosophical Studies. doi: 10.1007/s11098-017-0994-2

2. Cowling, S. (2013). Ideological parsimony. Synthese, 190(17), 3889-3908. doi: 10.1007/s11229-0120231-7

3. Elgin, S. Z. (n. d.). The Simplicity of Metaphysical Structures (Doctoral thesis). San Diego: University of California.

4. Parsons, T. (1979). The Methodology of Nonexistence. The Journal of Philosophy, 76(11), 649. doi: $10.2307 / 2025698$

5. Willard, M. B. (2013). Against simplicity. Philosophical Studies, 167(1), 165-181. doi: 10.1007/s11098-013-0228-1

6. Dilworth, C. (2012). Simplicity: A Meta-Metaphysics. Lanham: Lexington Books.

7. Kriegel, U. (2017). Metaphysics and Conceptual Analysis: Experimental Philosophy's Place under the Sun. In D. Rose (Ed.), Experimental Metaphysics (pp. 7-46). New York: Bloomsbury Academic.

8. Dowell, J. L. (2007). Serious metaphysics and the vindication of reductions. Philosophical Studies, 139(1), 91-110. doi: 10.1007/s11098-007-9104-1

9. Cornell, D. M. (2016). Taking monism seriously. Philosophical Studies, 173(9), 2397-2415. doi: 10.1007/s11098-015-0620-0

10. Paul, L. A. (2017). A One Category Ontology. Being, Freedom, and Method, 32-61. doi: 10.1093/acprof:oso/9780198715702.003.0003

11. Sider, T. (2013). Against Parthood. Oxford Studies in Metaphysics, 8, 236-293. doi: 10.1093/acprof:oso/9780199682904.003.0006

12. Inwagen, P. V. (1990). Material Beings. New York: Cornell University Press.

13. Miller, K. L. (2014). Defending Substantivism about Disputes in the Metaphysics of Composition. Journal of Philosophy, 111(9), 529-556. doi: 10.5840/jphil20141119/1036

14. Schneider, L. (2017). Minimal Aristotelian Ontology. Cosmos + Taxis, 4 (4), 27-37.

15. Brenner, A. (2016). Simplicity as a criterion of theory choice in metaphysics. Philosophical Studies, 174(11), 2687-2707. doi: 10.1007/s11098-016-0805-1

16. Markosian, N. (1998). Brutal Composition. Philosophical Studies, 92(3), 211-249. doi: 10.1023/a:1004267523392 